Document downloaded from:

http://hdl.handle.net/10251/56288

This paper must be cited as:

Velez, M.; Eizmendi, I.; David Gomez-Barquero; Morgade, J.; Baena Lecuyer, V.; Slimani, M.; Zoellner, J. (2014). DVB-T2: The Second Generation of Terrestrial Digital Video Broadcasting System. IEEE Transactions on Broadcasting. 60(2):258-271. doi:10.1109/TBC.2014.2312811.

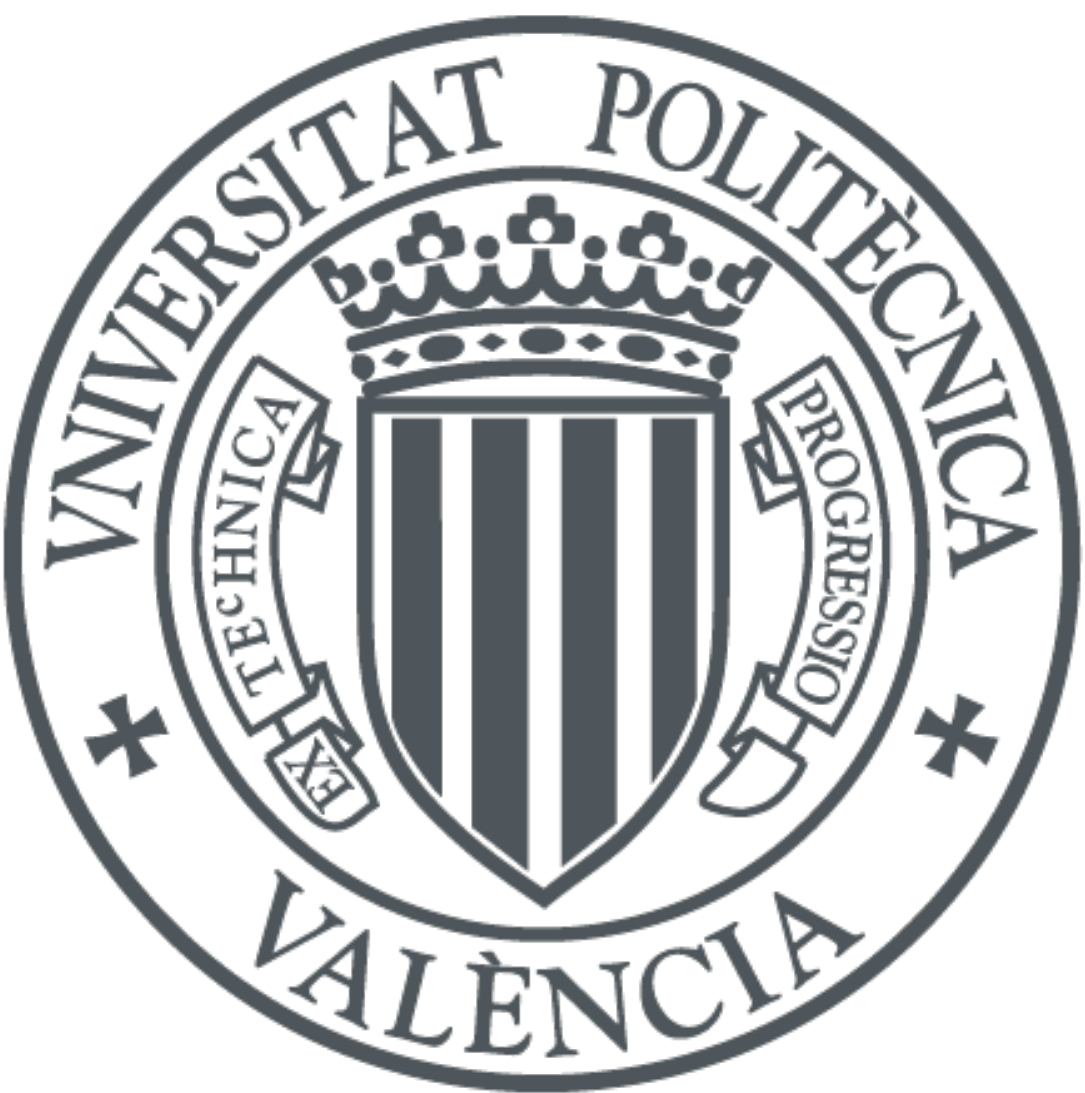

The final publication is available at

http://dx.doi.org/10.1109/TBC.2014.2312811

Copyright Institute of Electrical and Electronics Engineers (IEEE)

Additional Information 


\title{
DVB-T2, the Second Generation of Terrestrial Digital Video Broadcasting System
}

\author{
Iñaki Eizmendi, Manuel Velez, David Gómez-Barquero, Javier Morgade, Vicente Baena-Lecuyer, \\ Mariem Slimani, Jan Zoellner
}

\begin{abstract}
This paper provides a review of the second generation of Terrestrial Digital Video Broadcasting standard DVB-T2. DVB-T2 is the evolution of DVB-T and, together with DVB-S2 and DVB-C2, inaugurated a new transition from the first generation digital broadcasting systems, similar to the transition from analog to digital systems. In the paper the most relevant features of DVB-T2 are explained in detail, along with their benefits and trade-offs.

The paper also presents a comprehensive review of the laboratory and field trial results available so far. Especial emphasis is placed in the results of the measurements carried out to test the mobile reception and the novel technologies as Multiple Input Single Output (MISO) and Time Frequency Slicing (TFS).
\end{abstract}

Index Terms-Digital video broadcasting, Digital TV, DVB-T2, Network Planning.

\section{INTRODUCTION}

$\mathbf{I}$ N September 2009, ETSI published the first version of the DVB-T2 standard (V1.1.1), based on a DVB blue-book released about one year before. Two new versions have been published since then, V1.2.1 in February 2011, and V1.3.1 in April 2012. This last version is especially relevant because it introduces a new profile, named T2-Lite, intended to broadcast mobile services.

This standard for digital terrestrial television (DTT) was the second of a series of new generation digital television systems inside the DVB consortium. The first one was DVB-S2 and the third DVB-C2. In this way, DVB was able to augment its digital standards for satellite, terrestrial and cable TV with a family of new generation systems.

The aim of DVB in releasing the second generation was to introduce the developments achieved in signal processing since the first digital standards appeared in 1993. In 16 years new algorithms and signal processing techniques had been developed, and others already discovered for a long time were

Manuscript received xx xx, 2013; revised xx xx, 2014.

This work has been financially supported in part by the University of the Basque Country UPV/EHU (UFI 11/30), by the Basque Government (IT-683-13 and SAIOTEK), and by the Spanish Ministry of Economy and Competitiveness under the project HEDYT-GBB (TEC2012-33302).

Iñaki Eizmendi, Manuel Velez and Javier Morgade are with the Dpt. of Communication Engineering, Bilbao Faculty of Engineering, University of the Basque Country UPV/EHU, Spain (e-mail: inaki.eizmendi@ehu.es; manuel.velez@ehu.es; javier.morgade@ehu.es). David Gómez-Barquero is with the iTEAM Research Institute, Universitat Politècnica de València, Valencia, Spain (e-mail:dagobar@iteam.upv.es). Vicente Baena-Lecuyer is with the Dpt. of Electronic Engineering, Escuela Superior de Ingenieros, University of Seville, Seville, Spain (e-mail: baena@gte.esi.us.es). Mariem Slimani and Jan Zoellner are with Institut für Nachrichtentechnik (Institute for Communications Technology), Technische Universität Braunschweig, Braunschweig, Germany; (e-mail: slimani@ifn.ing.tu-bs.de; zoellner@ifn.ing.tu-bs.de) feasible because of the increasing processing capabilities of the hardware. The result is that the real performance of these new systems are now very close to the Shannon limit, which defines the limit of the efficiency for digital communications.

Spectral efficiency is becoming more and more important for DTT, because spectrum previously allocated for TV broadcasting is now being re-allocated to mobile communications systems, in particular 4G LTE. At the same time, quality requirements for TV are growing and HDTV is a must for people owning big flat-screen displays. On the other hand, the mass popularization of smart phones and tablets means that high quality displays from 4" to 10 " are carried in our pockets and bags ready to play digital content that, of course, can be sent by mobile data networks, but perhaps with the risk of saturation of those networks. Digital broadcasting should be able to provide this high bandwidth, demanding digital content.

DVB-T2 has been designed to fulfill these requirements, increasing spectral efficiency and robustness in a flexible way so that a variety of reception scenarios can be covered, with the same system by choosing the best configuration options available.

The remainder of this paper is organized as follows. The first part of the paper will be a comprehensive description of the system and a second part will describe the most relevant simulation, laboratory and T2 field trial results. Conclusions are drawn in Section IX.

\section{Motivation}

The motivation of DVB for the development of a new DTT standard can be found in the DVB Document A114, Commercial Requirement for DVB-T2, released in April 2007 [1] and that was used as a basis for DVB-T2 technology. Twenty one requirements were defined grouped in categories, such as transmission and receiving conditions, frequency efficiency, frequency bands and channel bandwidths, robustness, backwards compatibility, etc.

The main reason for launching DVB-T2 was the use of the new ways of modulating and error-protecting the broadcast stream to increase the efficiency in the use of radio spectrum. It was important to have this new technology ready when many countries had to perform the analog TV switch off and some others were adopting MPEG-4 as video coding technology to broadcast HD services. These changes in coding technology force the final user to buy a new TV set or a set top box (STB), so they were seen as a good opportunity to launch the new transmission standard. 


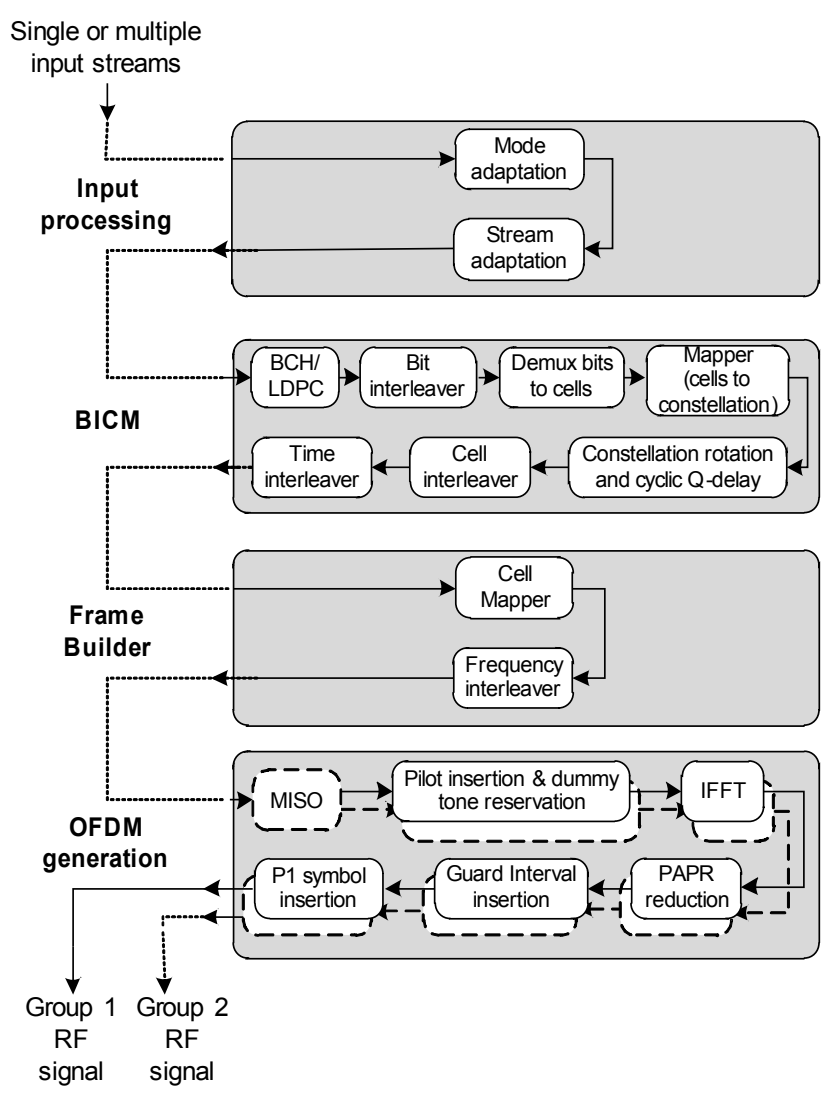

Fig. 1. DVB-T2 block diagram [2]

The main reception scenario for DVB-T2 was fixed reception, but portable and mobile reception were kept in mind from the beginning, as can be read in the first requirement [1]:

"The DVB-T2 specification shall be designed for stationary reception. However, it shall be possible to design DVB-T2 networks for all three receiving conditions, fixed, portable and mobile"

\section{SySTEM OVERVIEW}

Like many modern terrestrial broadcasting and radio communication systems DVB-T2 uses OFDM (orthogonal frequency division multiplex) modulation. DAB (Digital Audio Broadcasting) and DVB-T were in the nineties the first digital terrestrial broadcasting standards that made use of this technique. Since the introduction of the first DVB based DTT standards, many other wireless communications systems like IEEE 802.11, IEEE 802.16 and LTE have finally adopted OFDM as transmission technique.

As said before, the DVB-T2 standard was originated by the demands to increase the spectral efficiency of digital terrestrial systems in the UHF/VHF bands. The standard provides high flexibility in multiplex allocation, coding, modulation and RF parameters. The DVB-T2 transmission chain is depicted in Fig.1 where the main processing blocks are represented. Table I summarizes the new technologies included in each block where the main benefits are outlined.

Compared to DVB-T, DVB-T2 adds a new element in the architectural model, the T2-Gateway. This element is connected
TABLE I

BENEFITS

\begin{tabular}{c|l|c}
\hline Block & New Features & Benefits \\
\hline \hline & - PLP & Flexibility \\
Input & - New Input Formats & Flexibility \\
& - High Efficiency Modes & Less Overhead \\
\hline & - LDPC & Robustness \\
& - Capacity \\
BICM & - Rotated Constellations & Robustness \\
& - Time Interleaving & Robustness/Mobile \\
\hline Frame & - FEF & Flexibility \\
Builder & & Capacity \\
\hline \multirow{3}{*}{ OFDM } & - Larger FFT Sizes & Flexibility \\
Generation & - New Bandwidths & Better Performance/Mobile \\
& - Pilot Patterns & Energy efficiency \\
& - PAPR Reduction & Synchronization \\
\hline
\end{tabular}

to the modulator, or modulators in an SFN configuration, by an interface named T2-MI (T2 Modulator Interface) [3]. The T2-Gateway performs the needed tasks to ensure that all the modulators belonging to the same SFN generate the same signal, or the two possible signals in case of MISO SFN as introduced in section VII.

\section{A. Input Processing}

The DVB-T2 standard allows the following input formats:

- Transport Stream (TS). Stream with constant packet length, as in DVB-T.

- Generic Encapsulated Stream (GSE). Constant or variable length packets, where the format is known by the modulator. This format is intended to broadcast IP content without using TS-MPE (Multi-Protocol Encapsulation) [4].

- Generic Continuous Stream (GCS). Variable length packets. Modulator does not know the actual length.

- Generic Fixed-length Packetized Stream (GFPS). For compatibility with DVB-S2. Not expected to be used.

TS can still be used as in DVB-T. However, some optional mechanisms are designed to decrease the overhead that TS format introduces:

- Null Packet Deletion. Remove most of the null packets of the TS.

- High Efficiency Mode (HEM). Remove the SYNC (synchronization) byte of the TS. Also used with GSE format.

The receiver at the output will be able to replace the removed parts again. If TS format is going to be used, these options should be selected, because in general no drawbacks are present. These types of streams are allocated to T2 baseband frames (BBframes). Sometimes padding could be needed to adjust the input stream packets to the BBframes. Then the contents of the BBframe are scrambled.

\section{B. Physical Layer Pipes}

A remarkable improvement of DVB-T2 comes from the ability to define service specific robustness levels. With the 
Physical Layer Pipes (PLPs) different protection levels can be configured in DVB-T2, including the channel coding parameters, constellation orders and interleaving depths. A single DVB-T2 signal can therefore take one or more PLPs. This allows the system to be configured, for example, to carry two PLPs, one configured for high data rate (high order constellation and low protection) to broadcast one or more HD programs to be received by roof-top antennas, while the other one can be configured for high robustness with lower data rate in order to be received by portable or mobile receivers. Nevertheless, it should be taken into consideration that parameters related to the OFDM symbol configuration (such as FFT size and guard interval) are common to all PLPs.

\section{C. $B I C M$}

The BICM (Block Interleaving and Coding Modulation) includes all the interleaving, coding and modulation steps carried out over each BBframes of a given T2 PLP (Fig.1).

Acording to the BICM, the input BBframes of a PLP to be transmitted are first coded by an outer encoder $(\mathrm{BCH})$ and an inner encoder (LDPC).The use of the LDPC [5] is responsible for the robustness increase compared with other systems like DVB-T, and it is a common characteristic of the DVB second generation standard family (DVB-S2, DVB-T2 and DVB-C2) while the outer $\mathrm{BCH}$ encoder is intended to reduce the error floor of the LDPC [6]. Six code rates (CR) or protection levels are defined: $1 / 2,3 / 5,2 / 3,3 / 4,4 / 5$ and 5/6 (from more protected to less protected), and two sizes for the LDPC FEC frames, $16 \mathrm{~K}$ and $64 \mathrm{~K}$. Short FEC frames are slightly less robust (about $0.2 \mathrm{~dB}[7])$, but allow an easier scheduling, especially for low data rates.

Since the encoding scheme of DVB-T2 greatly outperforms the Convolutional and Reed Solomon codes used in DVB-T, DVB-T2 introduces the higher order constellation 256-QAM which increases the spectral efficiency and bit rate.

According to this structure the size of the BBframe (the input to the FEC frames) depends on the FEC frame sizes and the coding rate, because the FEC frames are fixed to $16 \mathrm{~K}$ and $64 \mathrm{~K}$. It should be also noted that in the DVB-T2 Lite specific profile two new code rates are added (1/3 and 2/5), and two are removed (4/5 and 5/6), and only short FEC frames are allowed.

The contents of FEC frames are then bit interleaved, except if QPSK constellation is going to be used, and finally mapped to constellations. The way the bits are grouped to form the constellation symbols adds some new interleaving. At this point, in the DVB-T2 standard, the term cell is used to reference the constellations points that will modulate the data carriers. A cell is defined by a complex value, I+jQ (In-phase and Quadrature-phase) samples.

One of the new features of DVB-T2 is the use of rotated constellations. Originally suggested in [8], this technique is also known as signal space diversity (SSD), since the final purpose is to lead to additional diversity that achieves a redundancy in information bits of the coded modulation. This solution improves the receiver performance when severely faded channels are encountered. When this feature is used,

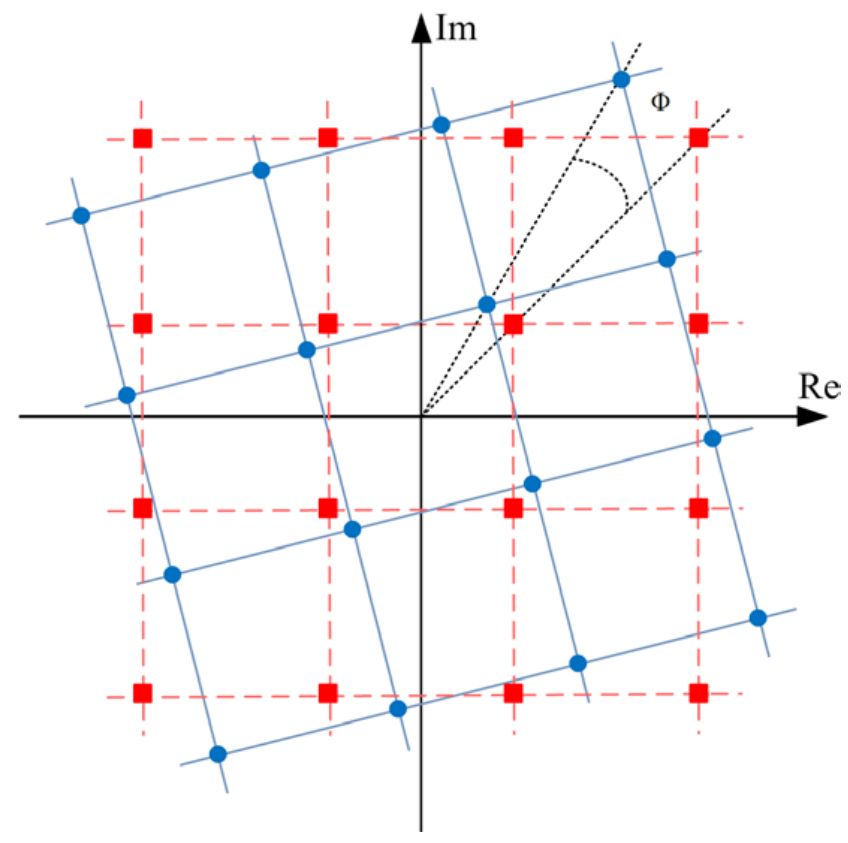

Fig. 2. Rotated and classic 16-QAM constellation. The square points represent the conventional constellation, whereas the circle points show the constellation points after rotating.

cells are rotated by a certain angle, as shown in Fig.2, where a rotated 16-QAM constellation and its corresponding conventional constellation are depicted. The rotation angle depends on the constellation used.

From Fig.2, it can be observed that due to this rotation, each new component, in-phase (I) or quadrature $(\mathrm{Q})$, has enough information by its own to determine which was the transmitted symbol. However, the use of rotated constellations does not offer by itself a remarkable improvement when both I and Q suffer from identical loss in the fading channel. To overcome this limitation, after the rotation, an interleaving process is performed between I and Q components to transmit both in different carriers and different time slots. This process is called Q-delay and ensures that both Q and I components of the original constellation point are finally transmitted in different $\mathrm{T} 2$ cells. This technique is know as constellation rotation and cyclic Q-delay (RQD).

The interleaving process guarantees therefore that both the I and Q components of the symbol are affected by independent fading. Thus, if one of the components is erased or affected by a deep selective fading of the channel, the other component can be used to recover the information [6]. Note that in a non-rotated constellation the information would be lost, since both components suffer the same fading when the signal is transmitted through the channel.

In conclusion, the rotated constellation technique introduces a higher degree of diversity to improve the DVB-T2 receiver performance, mainly in propagation scenarios with deep fading conditions or erasures events. Simulations [9], [10] show that the RQD technique provides a gain that can vary from $0.2 \mathrm{~dB}$ to several $\mathrm{dBs}$ depending on the order of the constellation, the $\mathrm{CR}$, and the channel model when compared to the conventional 
QAM constellations.

There is no capacity penalty in the use of this feature, but the complexity of the receiver is increased, especially for high order constellations. Some demapper algorithms have been developed in order to simplify this task [11], [12], [13]. In T2-Lite, to avoid this complexity, rotation is not allowed in combination with 256-QAM constellation.

After constellation mapping a new interleaving process is performed over the cells belonging to one FEC block. The interleaving sequence is different for each FEC block that will form the time interleaving frame.

The next to last interleaving process is the time interleaving. This interleaving is the longest one. It mixes cells coming from different FEC blocks. So it can be used to increase the robustness against low Doppler varying channels, but at the expense of increasing the zapping time. As time interleaving is a mandatory option, transmitters and receivers must be provided with the memory needed, regardless of whether time interleaving is used or not in the configuration. Time interleaving is performed in such a way that memory requirements in receivers are minimized.

The Time interleaving is performed at PLP level so each PLP can be interleaved in a different way and with different final interleaving time. For example a PLP intended for HD services to be received by roof-top antennas, does not need long interleaving times, but a PLP intended to be received by portable devices will be better protected if interleaving time is increased as much as possible, always keeping in mind that the zapping time is increased too.

The standard offers three different time interleaving options, depending on how FEC blocks, TI-blocks, interleaving frames, and T2-frames are related. The TI-blocks are the result of interleaving a number of FEC blocks, so the interleaving time depends on the length of the TI-blocks, but can be increased because the TI blocks can be split and allocated to several consecutive or non-consecutive T2-frames.

\section{Frame Builder}

This block is in charge of allocating the cells from the PLPs to the data carriers of the OFDM symbols, the OFDM symbols in T2-frames and finally, the T2-frames in the so called T2 super-frames. Also the signalling information has to be allocated in this structure.

The structure of the T2 frame begins with one special symbol, P1, intended for synchronization and signaling, followed by one or more $\mathrm{P} 2$ symbols, also used for signaling, and after that a number of data symbols (Fig.3). The number of data symbols is configurable, but the total length of the T2 frame must not exceed $250 \mathrm{~ms}$.

There are three different PLP types according to how they are allocated. Common PLPs carry information shared by other PLPs belonging to the same group (several groups can be defined) and they are allocated immediately after signaling.

Type 1 PLPs are allocated next. If one receiver is decoding one of these PLP, it can stop receiving data as long as the corresponding slice of the common PLP and of the decoded PLP are received, until the next T2 frame in which any of those PLPs are present. This way a portable the receiver can save battery.

Slices of type 2 PLPs are further divided, resulting a configurable number of sub-slices that are sent in a fixed sequence together with the rest of sub-slices of the other type 2 PLPs. This way the cells corresponding to these kind of PLPs are spread along the data symbols of the T2 frame, instead of being transmitted in some consecutive symbols, as happens with type 1 PLPs. Time diversity is increased, but at the expense of consuming more battery for reception, as the receiver needs to receive data more frequently.

The information on how PLPs are allocated in each T2 frame is part of the L1 signaling, so to be able to receive any PLP it is necessary to decode first this signaling.

The T2 superframes are composed of a number of T2 frames and optionally by one or more Future Extension Frames (FEFs). The part of the T2 superframe indicated as a FEF can be filled with any signal. The DVB-T2 receivers will ignore the signal during these periods of time.

For instance, the new version of DVB-T2 intended for mobile reception T2-Lite, can be sent together with DVB-T2 using this FEF mechanism. Moreover, the existence of FEFs provides a big flexibility to the system for new developments.

\section{E. OFDM Generation}

The last block of the DVB-T2 transmission chain is the generation of the OFDM symbols. DVB-T2 offers considerable flexibility in the OFDM symbol characteristics:

- Six FFT sizes: $1 \mathrm{~K}, 2 \mathrm{~K}, 4 \mathrm{~K}, 8 \mathrm{~K}, 16 \mathrm{~K}$ and $32 \mathrm{~K}$. (1K and $32 \mathrm{~K}$ are removed in T2-Lite)

- Six channel bandwidths: $1.7 \mathrm{MHz}, 5 \mathrm{MHz}, 6 \mathrm{MHz}$, $7 \mathrm{MHz}, 8 \mathrm{MHz}$ and $10 \mathrm{MHz}$.

- Seven Guard interval fractions: $1 / 128,1 / 32,1 / 16,19 / 256$, $1 / 8,19 / 128$ and $1 / 4$.

For the $8 \mathrm{~K}, 16 \mathrm{~K}$ and $32 \mathrm{~K}$ FFT sizes the extended carrier mode allows the use of more carriers per symbol which consequently increases the data capacity. Choosing the best parameters will depend on the application. In the case of mobile reception, the highest FFT sizes are not appropriate since the reduced carrier spacing limits the Doppler frequency that can be tolerated due to ICI (Inter Carrier Interference) but for example, for stationary reception, with large Single Frequency Networks (SFNs), in the UHF band and in ITU-R region 1 , the parameters could be: $32 \mathrm{~K}$ extended, $8 \mathrm{MHz}$, $19 / 128$ (1/4 is not possible in combination with $32 \mathrm{~K})$

In normal data symbols, carriers are used as pilot cells (continual, scattered or edge), data cells or dummy cells. The scattered pilots are used in the receiver for channel estimation, their distribution along the symbol follows eight different patterns. The use of less dense patterns results in lower overhead, and the use of denser ones results in more accurate channel estimation. Again it is necessary to know the intended reception scenario to choose the optimal option.

There is a special pilot pattern (PP8) with the lowest pilot density, that is intended to be used with a channel estimation algorithm named $\mathrm{CD} 3$, where the data cells of one symbol are used to obtain the frequency response of the following 


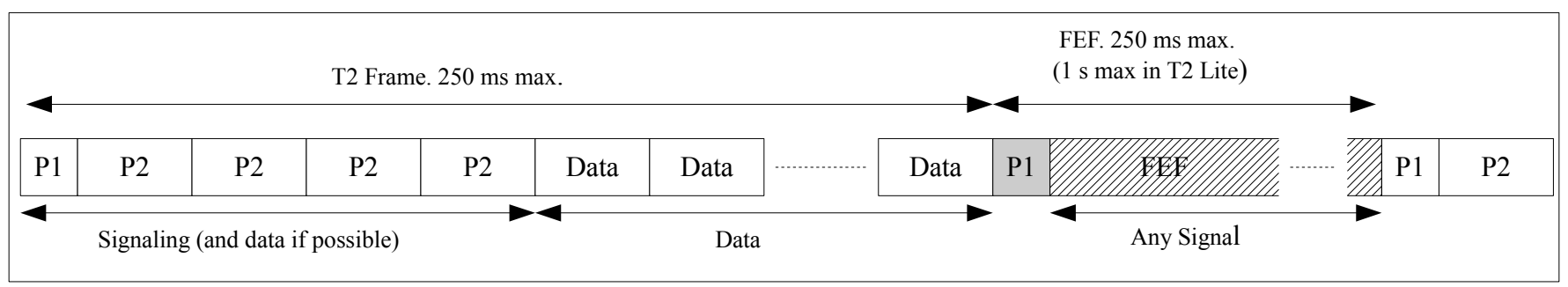

Fig. 3. T2 frame for $4 \mathrm{~K}$ FFT size (4 P2 symbols) and FEF

symbol(s) [14], [15]. This pilot pattern should not be used in combination with time interleaving or multiple PLPs [7].

Since one of the major drawbacks of orthogonal frequency division multiplexing is its large envelope fluctuations, the DVB-T2 standard includes two methods for peak-to-average power ratio (PAPR) reduction: Active Constellation Extension (ACE) [16] and Tone Reservation (TR) [17]. The use of these methods results in a back-off reduction of the high-power amplifier, increasing the power efficiency of the transmitter.

With TR some of the cells are not used as data cells, but their values are set such a way that the peak power of the OFDM signal is reduced. The penalty for the PAPR reduction is a reduction of about $1 \%$ in the system capacity.

ACE works modifying the value of cells using the outermost points of the constellation, again in such a way that the peak power is reduced. It cannot be used when rotated constellations are present, and in contrast with TR there is no capacity penalty when used.

Whereas the ACE method provides greater benefits in lower order constellations, the TR technique provides greater benefits in higher order constellations, although both can be applied simultaneously.

It is important to remember that the selected configuration of the OFDM symbol affects all the PLPs, so if different kinds of services are going to be broadcast a trade-off must be made to select a configuration suitable for all of them. The alternative is to use T2 and T2-Lite in the same RF channel using the FEF feature, allowing almost completely different configurations.

\section{F. Signaling}

Signaling information is divided into layer-1 (L1) and layer2 (L2) signaling. L1 signaling is related to the physical parameters of the signal and the way the information is organized whilst L2 is related to the data streams, for example the signaling of the MPEG-2 TS, and it is not specified in the DVB-T2 standard.

L1 signaling is further divided into L1-pre and L1-post. L1-pre provides static information about the frame structure and L1-post is mainly intended to provide information about how the information (PLPs) are sent. Two special symbols are used to transmit the L1-signaling, P1 and P2 symbols. The P1 symbol is sent at the beginning of each T2 frame and FEF. In $\mathrm{T} 2$ frames the P1 symbol is followed by one or several P2 symbols as in Fig.3. The P1 symbol is a kind of preamble, its configuration is the same however the rest of the system is configured. It is a $1 \mathrm{~K}$ OFDM symbol, with no cyclic prefix.

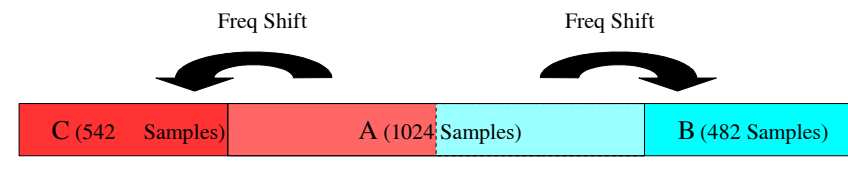

Fig. 4. P1 symbol time structure [2]

Instead, the symbol is repeated, with the repeated symbol split in two parts and separate frequency shifts applied to each part as shown in Fig.4.

The structure of the P1 symbol allows for a robust symbol detection and might be used for a coarse time synchronization of the received T2 signal [18], [19]. The P1 symbol can be detected even at about $-6 \mathrm{~dB} \mathrm{C} / \mathrm{N}$ (Carrier to Noise ratio) values [20]. Moreover, the frequency domain structure of the P1 symbol allows also for a fast frequency synchronization. Up to $500 \mathrm{~Hz}$ frequency offset can be detected in case of $8 \mathrm{MHz}$ nominal bandwidth. The P1 symbol is modulated with two fields (S1, 3 bits, and S2, 4 bits) that provide the following information: SISO/MISO, T2-Frame/FEF, FFT size, Guard Interval and FEF type. This information can be decoded also at negative $\mathrm{C} / \mathrm{N}$ values, and it is very useful for the fast reception of the rest of the symbols.

One or more $\mathrm{P} 2$ symbols follow the P1. The number is fixed for each FFT size and follows the rule: "number of symbol" times "FFT size (expressed in K)" equals 16 (except for 32K, for which it is 32), so for example for $1 \mathrm{~K}, 16 \mathrm{P} 2$ symbols are present. This way the capacity of the sum of all P2 symbols is always the same (again except for $32 \mathrm{~K}$, which doubles the capacity).

The P2 symbols transmit the rest of the L1 signaling and even data if there is some capacity left. The modulation and coding of L1 in P2 symbols are different from the rest of the system. BPSK $1 / 4$ is always used for L1-pre, and BPSK, QPSK, 16-QAM and 64-QAM, CR 1/2, can be selected for L1-post. It is important to configure L1-post robustness to be higher than the most protected PLP in the system, because if L1-post cannot be decoded, the PLPs will not be decoded either. But, as the capacity of P2 symbols is limited by the number of available cells, if a very robust constellation is selected the L1-post signaling could be larger than the available space and could not be allocated, in this case another constellation should be selected or the number of PLPs reduced, to reduce the amount of data in L1-post.

The use of the P2 symbols limits the time interleaving possibilities of the L1 signaling and has been seen as a weakness of DVB-T2 for mobile reception. 
TABLE II

MAIN CONFIGURATION OPTIONS

\begin{tabular}{c|l}
\hline Parameter & Values \\
\hline \hline Bandwidth $(\mathrm{MHz})$ & $1.7,5,6,7,8,10$ \\
FFT Size & $1 \mathrm{~K}^{1}, 2 \mathrm{~K}, 4 \mathrm{~K}, 8 \mathrm{~K}, 16 \mathrm{~K}, 32 \mathrm{~K}^{1}$ \\
Bandwidth extension & Yes/No (Allowed for 8K, 16K, 32K) \\
GIF & $1 / 128,1 / 32,1 / 16,19 / 256,1 / 8,19 / 128,1 / 4$ \\
Pilot Patterns & PP1, PP2, PP3, PP4, PP5, PP6, PP7, PP8 ${ }^{1}$ \\
PAPR Reduction & None, TR, ACE (Not allowed with rot. const.) \\
Constellations & QPSK, 16QAM, 64-QAM, 256-QAM \\
Rotation & Yes $^{2} / \mathrm{No}$ \\
L1 Modulation & BPSK, QPSK, 16QAM, 64-QAM \\
FEC size & $16 \mathrm{~K}, 64 \mathrm{~K} 1$ \\
Code Rate & $1 / 3^{3}, 2 / 5^{3}, 1 / 2,3 / 5,2 / 3^{2}, 3 / 4^{2}, 4 / 5^{1}, 5 / 6^{1}$ \\
Input Mode & $\mathrm{A}(\mathrm{Single}$ PLP), B (Multiple PLP) \\
Input Format & $\mathrm{TS}, \mathrm{GSE}, \mathrm{GCS}, \mathrm{GFPS}$ \\
\hline
\end{tabular}

1-Not allowed in T2-Lite

2-Not allowed for 256-QAM in T2-Lite

3-Only allowed in T2-Lite

TABLE III

CAPACITY EXAMPLES

\begin{tabular}{c|c}
\hline Configuration & Data Rate \\
\hline \hline $\begin{array}{c}\text { 1.7MHz, 4K FFT, 1/8 GIF, PP2 } \\
\text { L1 BPSK, L1 repetition, QPSK 1/3 }\end{array}$ & $764.7 \mathrm{kbps}$ \\
\hline $\begin{array}{c}\text { 8MHz, 4K FFT, 1/8 GIF, PP2 } \\
\text { L1 BPSK, L1 repetition, QPSK 1/3 }\end{array}$ & $3.90 \mathrm{Mbps}$ \\
\hline $\begin{array}{c}\text { 8MHz, BW ext., 32K FFT, 1/128 GIF, PP7 } \\
\text { L1 64-QAM, L1 repetition, 256-QAM 5/6 }\end{array}$ & $50.32 \mathrm{Mbps}$ \\
\hline
\end{tabular}

In order to increase the robustness of L1-post signaling, it can be repeated in two consecutive frames. In each frame the current L1-post signaling and the corresponding to the next frame can be sent.

In the first version of the DVB-T2 standard, the P2 symbol could theoretically have large bias and PAPR values if large numbers of PLPs were used, and some modifications have been introduced in the later versions to avoid these issues.

\section{G. Summary of Configuration Options}

Table II summarizes the configuration options that can be chosen to configure the system to fit the transmission and reception requirements. Time interleaving options have not been included because of the difficulty to explain all the possibilities in one table.

As can be seen, lots of options are available. Not all combinations are possible, for example only some combinations of FFT size, guard interval, and the pilot pattern are permitted [2], but even with these restrictions the number of combinations is still huge.

The capacity of the system will depend on some of these configuration parameters, and for an $8 \mathrm{MHz}$ channel, it ranges from 3.90 Mbps if QPSK 1/3 (T2 Lite) is used to 50.32 Mbps with 256-QAM 5/6 (Table III). Considering the $1.7 \mathrm{MHz}$ bandwidth for digital radio transmission (same bandwidth as $\mathrm{DAB})$ the minimum data rate is $765.77 \mathrm{kbps}$.
TABLE IV

C/N values $(d B)$ FOR T2-LITE NEW CODE RATES ACCORDING TO LABORATORY TESTS

\begin{tabular}{c|cc}
\hline MODCOD & Gaussian & Rayleigh \\
\hline \hline QPSK 1/3 & -1.0 & 0.8 \\
\hline QPSK 2/5 & 0.0 & 2.0 \\
\hline
\end{tabular}

\section{System Performance}

The system performance is provided in the implementation guidelines [7]. The EBU Technical Report 3348 [21] uses the values of the implementation guidelines to provide more elaborate values, adding some terms that are not completely specified in the guidelines.

In these documents several reception thresholds are provided for different constellations and protection levels and for four typical channel models in fixed reception, AWGN, F1 (Ricean), P1 (Rayleigh) and 0dB echo. These thresholds were obtained from simulations under perfect reception conditions and should be considered as lower limit for real thresholds that will never be overcome in a real reception situation.

Some simulation results are also provided for mobile reception, using TU6 channel at different Doppler levels and with several interleaving times

Finally, signaling is studied according to P1 symbol decoding and L1 pre and post signaling performance for AWGN and TU6 channels.

It must be noticed that simulation results for T2-Lite are not provided in the implementation guidelines. For the code rates already present in the base profile, the values of the guidelines could be used. For the new code rates of T2-Lite (1/3 and 2/5), the EBU report proposes the use of the DVB-S2 simulation values. Laboratory test results are shown in Table IV for QPSK with these new code rates [22].

\section{Planning Thresholds FOR FIXED RECEPTION}

Fixed reception is the main target of the system. The most suitable configurations are the ones that provide high data rate. The radio channel expected in this reception is not as bad as in the other reception scenarios, with limited multipath, almost stationary and generally with good signal level. The main cause of severe multipath could be the SFN configuration.

Under these circumstances high order constellations, large FFT sizes and least dense pilot patterns can be used. The effect of the time interleaving length should not be noticeable.

These kind of configurations have been tested to obtain realistic planning thresholds from field trials and laboratory test and to study the influence of some of the configuration parameters.

The field trials carried out in Spain during 2010 [23] provided $\mathrm{C} / \mathrm{N}$ threshold values for fixed reception for 256QAM and 64-QAM in combination with all coding rates, for different multipath levels from AWGN channel to $0 \mathrm{~dB}$ echo channel. The main parameters of the configurations tested are shown in Table $\mathrm{V}$

In these measurements the minimum $\mathrm{C} / \mathrm{N}$ value to receive 30 seconds of DVB-T2 signal without erroneous BBframes was obtained using a professional receiver. 
TABLE V

DVB-T2 SETTINGS FOR TESTED CONFIGURATION IN SPAIN IN 2010

\begin{tabular}{c|c|c|c|c|c}
\hline FFT & GIF & $\begin{array}{c}\text { BW } \\
(\mathrm{ext})\end{array}$ & $\begin{array}{c}\text { Pilot } \\
\text { Pattern }\end{array}$ & $\begin{array}{c}\text { Rotated } \\
\text { Const. }\end{array}$ & $\begin{array}{c}\text { Code } \\
\text { Rate }\end{array}$ \\
\hline \hline $32 \mathrm{~K}$ & $1 / 16$ & $8 \mathrm{MHz}$ & PP4 & Yes & all \\
\hline \multicolumn{6}{|c}{} \\
\hline $\begin{array}{c}\text { PAPR } \\
\text { Reduct. }\end{array}$ & $\begin{array}{c}\text { LDPC } \\
\text { Size }\end{array}$ & PLPs & $\mathrm{N}_{T I}$ & $\begin{array}{c}\text { TI } \\
\text { Type }\end{array}$ & $\begin{array}{c}\text { Input } \\
\text { Format }\end{array}$ \\
\hline \hline No & 64800 & 1 & 3 & 0 & TS HEM \\
\hline
\end{tabular}

TABLE VI

C/N MIN $(d B)$ ACCORDING TO SIMULATIONS AND IMPLEMENTATION LOSSES $(d B)$ FOR 64-QAM, 32K, 1/16, PP4

\begin{tabular}{cc|cc|cc|cc}
\hline $\begin{array}{c}\text { Code } \\
\text { Rate }\end{array}$ & Bitrate & \multicolumn{2}{|c|}{ Gaussian } & \multicolumn{2}{c|}{ Rician } & \multicolumn{2}{c}{ Rayleigh } \\
(Mbps) & C/N & Imp.L & C/N & Imp.L & C/N & Imp.L \\
\hline \hline $1 / 2$ & 20.69 & 9.9 & 1.3 & 10.2 & 1.2 & 11.9 & 1.5 \\
\hline $3 / 5$ & 24.87 & 12 & 1.0 & 12.3 & 1.1 & 14.0 & 1.6 \\
\hline $2 / 3$ & 27.67 & 13.5 & 0.8 & 13.8 & 0.9 & 15.6 & 1.8 \\
\hline $3 / 4$ & 31.13 & 15.1 & 0.8 & 15.4 & 1.2 & 17.7 & 1.9 \\
\hline $4 / 5$ & 33.21 & 16.1 & 0.9 & 16.6 & 1.0 & 19.2 & 2.2 \\
\hline $5 / 6$ & 34.62 & 16.8 & 0.8 & 17.2 & 1.1 & 20.2 & 1.9 \\
\hline
\end{tabular}

TABLE VII

C/N MIN $(d B)$ ACCORDING TO SIMULATIONS AND IMPLEMENTATION LOSSES $(d B)$ FOR 256-QAM, 32K, 1/16, PP4

\begin{tabular}{cc|cc|cc|cc}
\hline $\begin{array}{c}\text { Code } \\
\text { Rate }\end{array}$ & Bitrate & \multicolumn{2}{|c|}{ Gaussian } & \multicolumn{2}{c|}{ Rician } & \multicolumn{2}{c}{ Rayleigh } \\
(Mbps) & C/N & Imp.L & C/N & Imp.L & C/N & Imp.L \\
\hline \hline $1 / 2$ & 27.61 & 13.2 & 2.1 & 13.6 & 2.2 & 15.6 & 2.3 \\
\hline $3 / 5$ & 33.18 & 16.1 & 1.7 & 16.3 & 2.1 & 18.3 & 2.4 \\
\hline $2 / 3$ & 36.92 & 17.8 & 1.4 & 18.1 & 1.7 & 20.1 & 2.6 \\
\hline $3 / 4$ & 41.53 & 20.0 & 1.4 & 20.3 & 2.0 & 22.6 & 3.3 \\
\hline $4 / 5$ & 44.32 & 21.3 & 1.5 & 21.7 & 2.5 & 24.3 & 4.2 \\
\hline $5 / 6$ & 46.20 & 22.0 & 1.7 & 22.4 & 2.4 & 25.4 & 3.5 \\
\hline
\end{tabular}

The measured $\mathrm{C} / \mathrm{N}$ thresholds can be used to calculate the implementation losses (threshold increment from implementation guidelines values to field measurements results). As said before, simulated thresholds were obtained assuming perfect reception conditions, that is, perfect channel estimation and synchronization, and genie-aided demapping [7] which are all impossible to reach in real receivers. Hence the implementation margin accounts mainly for the practical implementation of these aspects.

Tables VI and VII show the implementation losses for 64QAM and for 256-QAM. As can be seen, they are in a range of:

- 0.8-2.1 dB for Gaussian channel

- 0.9-2.5 dB for Ricean channel

- 1.5-4.2 dB for Rayleigh channel

During the year 2011 some measurements were done in Finland to test the performance of rotated constellations. Both, laboratory tests and field trials were performed [24]. Two kinds of measurements were done:

- Rotated Constellation Gain (RCG) vs. Channel Models
(Laboratory and field)

- RCG vs. Co-Channel Interference (Only Laboratory)

In both cases the DVB-T2 signal was impaired and the robustness was compared with and without rotated constellations.

The results showed that rotated constellations increase the robustness when Co-Channel Interference is present, allowing a larger part of the spectrum to be interfered with, but in the case of multipath, differences were only noticeable for large degrees of multipath and in some cases the performance of rotated constellations was poorer than normal constellations.

Similar results were obtained by laboratory and field results carried out in Spain [23] [25].

These tests were performed using commercial receivers, and it is unknown if the reception algorithms were optimized to take full advantage of constellation rotation diversity.

\section{Mobile Performance}

Although DVB-T2 primarily targets static and portable reception, it also incorporates time interleaving in order to benefit from time diversity in mobile scenarios. In addition to time interleaving, other improvements introduced in DVB-T2 that enable a higher robustness in mobile scenarios compared to DVB-T are: better FEC that improves the overall system performance in static and mobile channels, rotated constellations that improve the system performance in fading channels, and distributed Multiple-Input Single-Output (MISO) for improving the reception in SFNs.

DVB-T2 also supports the transmission of fixed/portable and mobile services in the same frequency channel by means of multiple PLPs. Furthermore, the mobile profile T2-Lite provides improved support for transmitting fixed and mobile services simultaneously. In particular, T2-Lite and the FEF feature allows a DVB-T2 signal optimized for fixed reception, together with a T2-Lite signal optimized for mobile reception, to be combined in the same T2 multiplex.

Mobile reception has been tested in several studies, field trials and laboratory tests. The variety of situations and the non-stationary mobile channel make the study of mobile reception more difficult than fixed reception, and reception thresholds are not easily obtained. Nevertheless it is important to analyze the influence of some configuration parameters and the robustness of the system against the propagation effects in mobile reception, like Doppler spread and fading.

\section{A. Time Interleaver}

In DVB-T2 a time interleaver was included at the physical layer in order to combat impulsive noise and benefit from time diversity in mobile scenarios. The time interleaver consists of a block interleaver that operates on a cell level. Each cell represents a complex symbol that is transmitted in a different subcarrier within the Orthogonal Frequency Division Multiplexing (OFDM) symbols. The time interleaver can be configured on a service basis and can provide interleaving durations ranging from few milliseconds up to several seconds. The interleaver does not support fast zapping in the sense that receivers have to wait until the complete reception of 
TABLE VIII

MAXIMUM TOLERABLE DOPPLER FREQUENCY $(\mathrm{Hz})$ FOR DIFFERENT MODCOD AND FFT SIZES

\begin{tabular}{c|cc|cc|cc}
\hline FFT & \multicolumn{2}{|c|}{ QPSK } & \multicolumn{2}{c|}{ 16QAM } & \multicolumn{2}{c}{ 64-QAM } \\
Size & $\mathbf{1 / 2}$ & $\mathbf{2 / 3}$ & $\mathbf{1 / 2}$ & $\mathbf{2 / 3}$ & $\mathbf{1 / 2}$ & $\mathbf{2 / 3}$ \\
\hline \hline $2 \mathrm{~K}$ & 296 & 331 & 173 & 149 & 169 & 128 \\
\hline $4 \mathrm{~K}$ & 166 & 142 & 112 & 88 & 73 & 56 \\
\hline $8 \mathrm{~K}$ & 63 & 55 & 48 & 39 & 32 & 22 \\
\hline $16 \mathrm{~K}$ & 36 & 30 & 24 & 17 & 15 & 10 \\
\hline $32 \mathrm{~K}$ & 21 & 13 & 12 & 8 & 7 & 5 \\
\hline
\end{tabular}

one time interleaving block before they can de-interleave and process the FEC blocks. The longer the interleaving duration, the longer the receivers must wait prior to the de-interleaving. The average channel change time is approximately 1.5 times the interleaving duration and therefore, long time interleaving in DVB-T2 cannot be provided at the physical layer with tolerable channel change times. The time interleaver is very flexible and it allows different trade-offs in terms of transmission robustness (time diversity), latency and power saving. A detailed study of the trade-offs in the context of mobile reception can be found in [26]

\section{B. FFT Size}

One of the main parameters related to the mobile performance is the FFT size due to its relation with the influence of Doppler spread. Smaller FFT size improves the performance, but at the expense of spectral efficiency, because for the same guard interval duration the guard interval fraction of the useful part is larger.

To test the influence of the Doppler frequency in the reception of DVB-T2, laboratory tests were performed in Spain. The DVB-T2 signal was generated and a TU6 channel was simulated with variable Doppler frequency. A professional $\mathrm{T} 2$ receiver was used, and the maximum Doppler frequency at which the signal was received without BBframe errors for 30 consecutive seconds was measured [27]. The results are shown in Table VIII. These results were obtained using pilot pattern PP2 because, as shown in next subsection it is the one that performs best.

Some Doppler frequency examples at an RF channel frequency of $600 \mathrm{MHz}$ are:

- For urban traffic speed, $40 \mathrm{~km} / \mathrm{h}$ or $25 \mathrm{mph}, 22 \mathrm{~Hz}$. In this case $8 \mathrm{~K}$ FFT or even $16 \mathrm{~K}$ could be used.

- For highway speed, $130 \mathrm{~km} / \mathrm{h}$ or $80 \mathrm{mph}, 72 \mathrm{~Hz}$. $4 \mathrm{~K}$ should be used.

- For high speed trains, $250 \mathrm{~km} / \mathrm{h}$ or $155 \mathrm{mph}, 139 \mathrm{~Hz} .4 \mathrm{~K}$ can still be used but with MODCOD QPSK 2/3 or higher protection, or $2 \mathrm{~K}$ should be selected.

\section{Pilot Patterns}

Apart from the FFT size the other main OFDM related parameter that most influences the mobile performance is the Scattered Pilot Pattern. In the same tests [27] several pilot patterns were tested and the results showed that pilot
TABLE IX

MAXIMUM TOleRABle DOPPLER FREQUENCY $(H z)$ FOR 16QAM $1 / 2$ AND ALL ALLOWED FFT SIZES AND PILOT PATTERN COMBINATIONS

\begin{tabular}{c|ccccc}
\hline Pilot & \multicolumn{5}{|c}{ FFT Size } \\
Pattern & 2K & 4K & 8K & $\mathbf{1 6 K}$ & 32K \\
\hline \hline PP1 & 128 & 59 & 27 & 13 & - \\
\hline PP2 & 173 & 112 & 48 & 24 & 12 \\
\hline PP3 & 101 & 42 & 21 & 11 & - \\
\hline PP4 & 200 & 93 & 40 & 20 & 9 \\
\hline PP5 & 71 & 32 & 15 & 8 & - \\
\hline PP6 & - & - & - & 14 & 7 \\
\hline PP7 & 72 & 31 & 13 & 7 & 4 \\
\hline
\end{tabular}

patterns numerated with even numbers (e.g. PP2, PP4 and PP6) performed better than the ones with odd numbers that add the same overhead (e.g. PP2 better than PP1 for 8,33\% overhead). As example, Table IX shows the results for 16-QAM 1/2.

The reason for this is that in even numbered pilot patterns the separation between pilots in the time direction (named $D_{y}$ in the standard) is 2 while in odd numbered ones $D_{y}$ is 4 , so the later are more sensitive to fast channel variations. The exception to this rule is the special case PP8 for which $D_{y}$ is 16.

\section{Results from the field trial in Northern Germany}

This section introduces briefly the DVB-T2 field trial in Northern Germany including a short description of the trial network and the measurement route. Afterwards, the most important results for the mobile performance are presented. A detailed presentation of all the measurement results can be found in [28].

The DVB-T2 field trial in Northern Germany [29] took place from August 2009 to July 2012 in the region south of Hamburg. The purpose of this trial was to define a potential introduction strategy of DVB-T2 for Germany. The investigations focused on the following scenario: broadcasting to portable and mobile receivers that are only equipped with small, non-directional antennas.

The trial network comprised two transmitters, which were about $45 \mathrm{~km}$ apart and operated at $690 \mathrm{MHz}$ in an SFN mode. The measurement route for the evaluation of the DVBT2 mobile performance comprised different areas situated between both transmitters and having different reception characteristics. These areas include cities and town centers with heavy traffic, dense buildings and a maximum allowed speed of $50 \mathrm{~km} / \mathrm{h}$, as well as country roads (up to $100 \mathrm{~km} / \mathrm{h}$ ) and one German "Autobahn" which has no speed limit. To analyze the mobile measurements, no test receivers were available on the market. Therefore, a mobile DVB-T2 measurement receiver [30] was developed by the Institut fuer Nachrichtentechnik of Technische Universitaet Braunschweig, which is based on "a software-defined-radio" concept. In addition to the decoding of the received signal, the receiver is also able to determine the characteristics of the transmission channel and to calculate a variety of measurement parameters. 


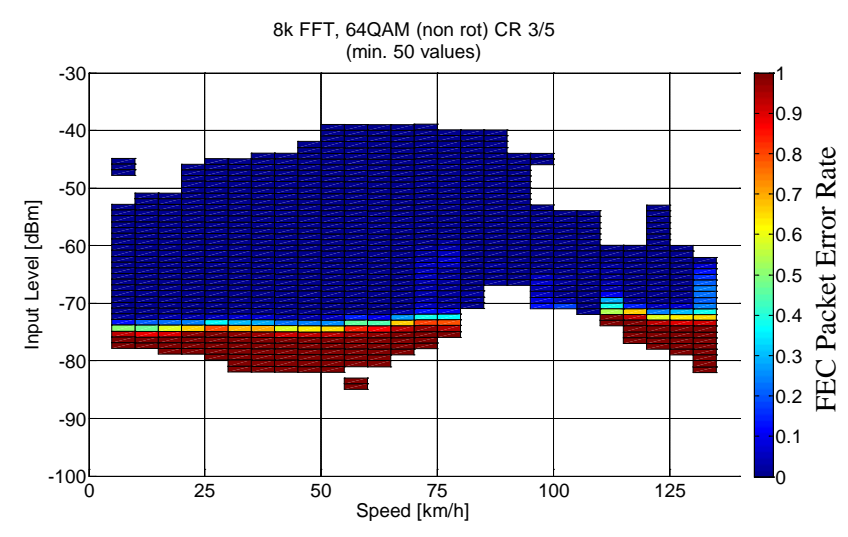

Fig. 5. BCH error rate as a function of the input level and the driving speed. 8k FFT, GI 1/4, PP1, and 64-QAM CR 3/5.

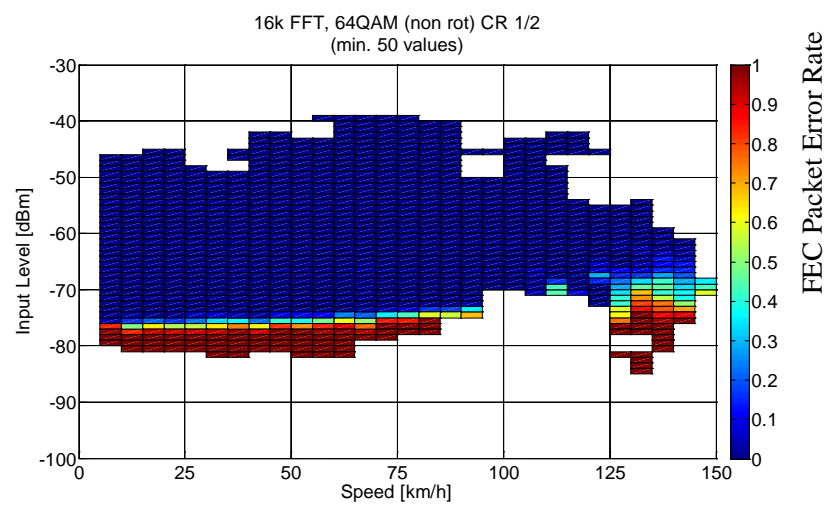

Fig. 6. $\mathrm{BCH}$ error rate as a function of the input level and the driving speed. 16k FFT, GI 19/128, PP2, and 64-QAM CR 1/2.

1) FFT Size: DVB-T works very well at high receiver velocities, as approx. 1 million cars in Germany are already equipped with DVB-T receivers that successfully receive the DVB-T signal with the $8 \mathrm{k}$ mode. The DVB-T2 field trial in Northern Germany focused on the $16 \mathrm{k}$ and the $32 \mathrm{k}$ mode, which would reduce the overhead caused by the Guard Interval of the OFDM modulation. The field measurements showed different results in comparison to the laboratory tests presented above. Fig.5, 6 and 7 show the results of three measurements using different FFT sizes. The first measurement used 8k FFT (Fig.5), the second one 16k FFT (Fig.6) and the third one 32k FFT (Fig.7), all with 64 QAM but with different LDPC code rates. Each diagram is a three-dimensional presentation of the FEC packet error rate as a function of the driving speed and the input level. The diagrams show that the 8k FFT and the 16k FFT allow for a good reception even with higher speeds of the receiver the $32 \mathrm{k}$ FFT, however, is only suitable until about $50 \mathrm{~km} / \mathrm{h}$.

2) Rotated Constellations: As mentioned in the beginning of this section, DVB-T2 introduced Rotated Constellations as one innovative algorithm to increase the robustness of the signal in strong frequency selective channels. In order to evaluate the performance of Rotated Constellations in a mobile environment, field measurements were carried out using a variety of QAM/FEC combinations, each with non-

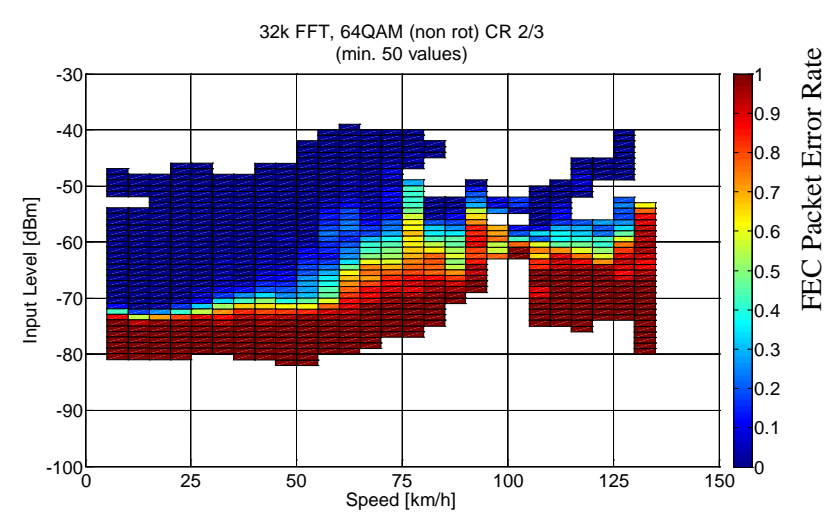

Fig. 7. $\mathrm{BCH}$ error rate as a function of the input level and the driving speed. 32k FFT, GI 1/16, PP2, and 64-QAM CR 2/3.

rotated and with Rotated Constellations. Only the QAM/FEC combination 16-QAM CR 3/4 offered a significant gain using Rotated Constellations, all other combinations showed a loss in comparison to non-rotated constellations. However, the 16QAM CR 3/4 mode should not be used. Based on other field tests, this mode requires a higher $\mathrm{C} / \mathrm{N}$ for error free reception than 64-QAM CR 1/2, although both modes offer the same spectral efficiency.

3) Pilot Patterns: Pilot pattern PP2 and PP3 were investigated in the mobile field tests in Northern Germany. Different QAM/FEC combinations with 16k FFT were used. The results showed, that both pilot patterns revealed a similar $\mathrm{C} / \mathrm{N}$ performance at a low speed. However, there was a clear difference with higher speeds. Only pilot pattern PP2 ensured undisturbed reception at higher speeds. This confirms the results of the laboratory tests mentioned in section VI-C

\section{E. DVB-T2 LITE}

T2-Lite is a mobile profile that was added to the DVB-T2 specification in release 1.3.1. T2-Lite is a better option than using multiple PLPs to multiplex fixed and mobile services in the same RF channel. In the case of multiple PLPs, the FFT mode and the pilot pattern must be common for all the PLPs transmitted in the same multiplex, whereas the T2-Lite profile allows alternating different configurations in the time domain by means of FEF parts.

The T2-Lite profile targets exclusively mobile and handheld receivers and thus, it contains only the transmission modes that are suited for mobile reception while minimizing the amount of receiver complexity. The profile was designed in order to reduce the complexity of T2-Lite-only receivers so as to minimize the cost and power consumption of handheld devices. For example, it establishes restrictions in terms of time interleaver memory, service data rate and FEC processing rate. On the other hand, the number of new elements in T2-Lite has been restricted in order to retain the maximum compatibility. In particular, the new profile incorporates more robust code rates to enable the reception at lower $\mathrm{C} / \mathrm{N}$, and also allows a higher flexibility for the multiplexing of different $\mathrm{T} 2$ signals in the same frequency channel.

Thanks to the Future Extension Frame (FEF) feature of DVB-T2, it is possible to introduce a T2-Lite signal optimized 
TABLE X

CONFIGURATION FOR THE COMBINATION OF FIXED AND MOBILE SERVICES IN DVB-T2 NETWORKS

\begin{tabular}{c|cc|cc}
\hline \multirow{2}{*}{$\begin{array}{c}\text { Configuration } \\
\text { Parameter }\end{array}$} & \multicolumn{2}{|c|}{ Configuration based } & \multicolumn{2}{c}{ Configuration based } \\
on mixed & Mobile & \multicolumn{2}{c}{ Fixed } & Mobile \\
\hline \hline FFT size & $8 \mathrm{~K}$ Ext. & $8 \mathrm{~K}$ Ext. & $32 \mathrm{~K}$ Ext. & $8 \mathrm{~K}$ Ext. \\
\hline Guard interval & $1 / 4$ & $1 / 4$ & $1 / 16$ & $1 / 4$ \\
\hline Pilot pattern & PP1 & PP1 & PP4 & PP1 \\
\hline Constellation & $256-\mathrm{QAM}$ & QPSK & $256-\mathrm{QAM}$ & QPSK \\
\hline Code rate & $3 / 5$ & $1 / 2$ & $3 / 5$ & $1 / 2$ \\
\hline
\end{tabular}

for mobile reception inside a T2 multiplex. A T2-Lite signal can occupy an entire frequency channel or can be multiplexed with other signals by means of FEF parts. The combination of T2-Lite with DVB-T2 transmissions is expected to be the first manner in which commercial T2-Lite services will be transmitted over the air. For example, it would be possible to dedicate $80 \%$ of the transmission time to DVB-T2 and $20 \%$ to T2-Lite. Assuming that the T2-Lite signal is transmitted with FFT size $8 \mathrm{~K}$ (with extended carrier mode), QPSK1/2, and pilot pattern PP1, the total capacity for T2-Lite services is approximately $1 \mathrm{Mbps}$ per channel (8 MHz bandwidth). This would allow up to 3 services at about $365 \mathrm{kbps}$ to be carried in the T2-Lite signal.

Table $\mathrm{X}$ shows an example comparing multiple PLPs and T2-Lite to transmit fixed and mobile services. The first configuration is based on multiple PLPs and employs a robust combination of FFT size and pilot pattern for the entire multiplex. In contrast, the second configuration is based on T2-Lite and alternates between different combinations for the transmission of fixed and mobile services.

In the table, it can be seen that both configurations provide the same robustness for fixed and mobile services and also the same duration of guard interval (SFN distance). However, with T2-Lite it is possible to use for fixed reception a more suitable FFT size and pilot pattern, which results in a higher spectral efficiency. In particular, the GI overhead can be reduced from $1 / 4$ to $1 / 16$ while keeping the same SFN distance when using an FFT $32 \mathrm{~K}$ instead of $8 \mathrm{~K}$, and also the pilot overhead is decreased using a less dense pilot pattern (in the example from PP1 down to PP4). The overall spectral efficiency gain depends on the percentage of time dedicated to the transmission of mobile services.

For example, if the percentage of resources devoted to mobile services is $20 \%$ ( $1 \mathrm{Mbps}$ as explained before), the available capacity for fixed services would be $21.5 \mathrm{Mbps}$ with multiple PLPs, and 26.7 Mbps with T2-Lite.

DVB-T2 lite measurements were done in Barcelona (Spain) in 2011. Some results are shown in [31] for indoor pedestrian measurements. The receiving location was the exhibition center of Barcelona.

The results show the possibility of transmitting both DVB-T2 and DVB-T2 Lite signals and the improvement obtained when the T2 Lite signal is properly configured for mobile portable indoor reception ( $4 \mathrm{~K}$ FFT size), while the DVB-T2 signal is configured for fixed reception (8k FFT size). For the same transmitting power $(1 \mathrm{~W})$ the percentage of correct reception grows from $43.7 \%$ for DVB-T2 to $67.5 \%$ for DVB-T2 Lite.

\section{DVB-T2 MISO}

Compared to Multiple Frequency Network (MFN) operation, an SFN will theoretically deliver the same quality with a certain amount of reduction in the transmitter power. This assumption is based on the fact that the receiving field strength will be more homogeneously distributed due to the spatial diversity associated to SFN networks. Nevertheless, the accumulated experience from the first generation terrestrial broadcast systems like DVB-T, has shown that some areas of a SFN will also present degradation caused by the reception of multiple echoes from different transmitters. The number of transmitters, the relative delay, as well as the power imbalance between received paths will have an impact on the final shape of the service area.

A significant amount of standardization effort during the development of DVB-T2 was therefore focused on the improvement of the Single Frequency Network (SFN) operation. In DVB-T2, new diversity mechanisms like Multiple Input Single Output (MISO) antenna diversity have been defined. A DVB-T2 MISO network should be viewed therefore as a particular form of an SFN, as the multiple transmissions require synchronization and timing as in traditional SFN. One benefit of this DVB-T2 MISO is that it can be implemented with a little additional complexity at both the transmit and received sides, this is important because all receivers should be able to receive a MISO signal, as it is a mandatory option. Nevertheless, there are some constrains inherent to the system design that restrict the available choice of guard interval and pilot pattern. For instance, in MISO mode, guard inteval 1/4 is not available and it is only suitable for networks designed with guard intervals up to about 19/128.

\section{A. Factors affecting the SFN-SISO vs SFN-MISO operation}

The diversity scheme used in DVB-T2 is based on the methodology described by Alamouti in [32] using a 2x1 STBC (Space Time Block Code) antenna group diversity scheme. However, the methodology adopted by DVB-T2 differs from the original Alamouti scheme in a way that payloads cells are processed in the frequency domain as shown in [2] [7]. The basic procedure relies on the fact that signals transmitted from transmitters that belong to MISO group1 are transmitted without any additional processing; nevertheless, signals in MISO group2 are pair wise modified.

Different factors can influence the practical SFN operation. A priori, the key factors are: Power imbalance, relative delay, propagation channel, pilot pattern, modulation and code rate. Those parameters are relevant factors in both traditional SISO and MISO procedures. However there might be differences in how each factor impacts the final network behavior.

\section{B. Related research activities}

This section describes a summary of the reference results available in relation to MISO in DVB-T2. These references 


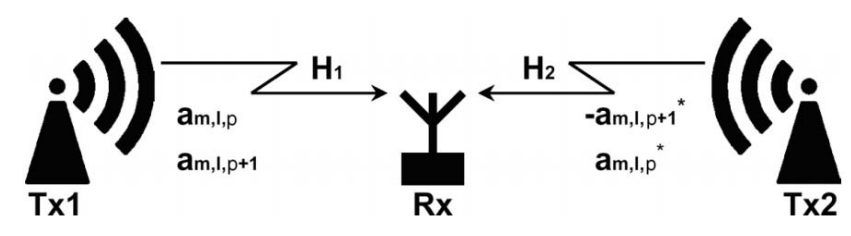

Fig. 8. DVB-T2 Distributed MISO [2]

can be divided into three sub-sets. The first one deals with the different simulations and field trials that have been carried out in France. The second one comprises the field trial carried out in northern Germany and finally the last sub-set deals with the simulations and the DVB-T2 distributed MISO measurement campaign of southern Germany.

The first set of results has been published by TDF France and the Universite Paul Verlaine in Metz. Most of the results by these authors [33] are based on simulations of different modes, reception scenarios and channel models proposed in the DVB-T2 implementation guidelines. The authors have analyzed the impact of power imbalance and relative delay between MISO components. The simulations provide MISO gain values for a TU6 propagation channel and assume perfect channel estimation stages. Simulations are therefore appropriate for a mobile reception characterization. The DVB-T2 modes selected are also suited for this type of reception. MISO gain values that range from -0.6 to $0.8 \mathrm{~dB}$ were obtained depending on the DVB-T2 mode and other factors. The authors identified the power imbalance between MISO components as the key factor. The results showed that power imbalance values lower than $6 \mathrm{~dB}$ provide positive MISO gains, whereas imbalance values higher than $6 \mathrm{~dB}$ present certain degradation. Recently, the same authors have carried out field experiments in Metz [34]. This trial consisted of measurements within the coverage area of a distributed MISO network of two transmitters. The data provided are mainly appropriate for mobile reception, where the major conclusion from this trial is that the performance of the equivalent SISO mode was better than MISO. However, the authors remark that the results might be influenced by a lack of adequate channel estimator for mobile reception in the evaluated $\mathrm{T} 2$ receiver.

The second and third set of results have been collected from field trials in northern and southern Germany. Reference results from the northern trial can be found in [35] where DVB-T2 MISO gain results are presented. The authors propose a method to estimate the MISO gain based on a gain predictor calibrated independently with real data collected. As with the previous reference, the authors state the power imbalance as the key factor affecting the MISO gain.

The final set of results available so far are the results from the southern Germany DVB-T2 MISO field trial in Munich. This trial was carried out during the summer of 2012 and results are collected in [36]. Provided results accomplish two complementary approaches based on empirical data and system level simulations to analyze the practical performance of DVB-T2 in two equivalent SFN-SISO and SFN-MISO configurations. In this work the dependency of the power imbalance is evaluated, but in addition the influence of the
TABLE XI

Measured EmPirical Miso Gain: 64QAM 2/3 LDPC 64800, 32KFFT, GI 1/16, PP2

\begin{tabular}{c|cc}
\hline & \multicolumn{2}{|c}{ MISO Gain (dB) } \\
Channel Profile & T2-Rx1 & T2-Rx2 \\
\hline \hline SFN & 1.84 & 1.13 \\
\hline Rician & 0.32 & -0.13 \\
\hline Rayleigh & -0.76 & -0.32 \\
\hline
\end{tabular}

TABLE XII

MeAsured Empirical Miso GaIN: 64QAM 3/4 LDPC 64800, 32KFFT, GI 1/16, PP2

\begin{tabular}{c|cc}
\hline & \multicolumn{2}{|c}{ MISO Gain (dB) } \\
Channel Profile & T2-Rx1 & T2-Rx2 \\
\hline \hline SFN & 2.45 & 1.58 \\
\hline Rician & 0.17 & 0.25 \\
\hline Rayleigh & -0.85 & -0.13 \\
\hline
\end{tabular}

channel characteristics is also taken into account. This last factor has been proven to be critical on the overall MISO performance.

\section{Performance margins}

From the contributions mentioned above, it has been proved that the practical DVB-T2 MISO performance in a distributed scenario is mainly limited by the available margins in terms of power imbalance between MISO groups, being therefore the available gain margins in DVB-T2 MISO limited to the transmitter SFN overlapping areas. The collected $\mathrm{C} / \mathrm{N}$ thresholds in MISO show that a valuable gain can be achieved in hard SFN channel conditions however current available references also state that a certain degradation might happen in MISO compared to the traditional SISO case in SFN scenarios that lead to high power imbalance margins between MISO antenna groups. The influence of the code rate is also addressed in most references, the achievable MISO gain being higher with lower robustness of the DVB-T2 mode. Some authors have also observed that the choice of receiver influences the overall MISO performance. Results from [34] and [36] have addressed a strong influence of the receiver implementation. This influence can be more evident for SFN scenarios with high relative delays between MISO groups where the lack of reference pilots resolution for equivalent SISO and MISO configurations can lead to a performance degradation in large SFN scenarios. Tables XI and XII outline some empirical MISO margins collected during the DVB-T2 MISO field trials in Munich for an equivalent SISO and MISO configuration [36]. Two different DVB-T2 target receivers were evaluated where $\mathrm{T} 2-\mathrm{Rx} 1$ is a state of the art commercial DVB-T2 receiver and T2-Rx2 represents a custom software defined radio DVB-T2 receiver implementation.

\section{DVB-T2 Time Frequency SLICING}

Time Frequency Slicing (TFS) is one of the novel tools available as an option in the DVB-T2 standard [2]. In TFS 
multiple RF channels can be combined into a wider virtual channel where in addition to the augmented overall bit rate, an improvement of the system frequency diversity can be achieved. TFS in not part of the "single profile" and it is referred to in the standard as future implementation in an informative annex. Nevertheless, the current available specification in [2] details all the required steps to support the TFS profile.

\section{A. The DVB-T2 Time Frequency Slicing Profile}

Time-Frequency-Slicing (TFS) is a DVB-T2 profile where the sub-slices of a PLP are sent over multiple RF frequencies during the $\mathrm{T} 2$-frame. In addition of the common interleaving process applied in single profile, with TFS interleaving might be also applied over multiple (not necessarily adjacent) RF channels.

As already described, DVB-T2 has two different types of PLPs depending on the number of time slices per frame configured in the given T2 system. PLPs type 1 are always carried using a single time slice per frame, on the other hand PLPs type 2 can be sent over multiple sub-slices. DVB-T2 also defines common PLPs, containing information associated to other PLPs that share the same group Id [2].

In TFS the T2-frame has a similar structure as the traditional single RF channel signal. However, in TFS there will be some restrictions in the frame length to enable enough time for switching between the different RF channels that convey the single TFS multiplex. Regarding the transmitted information, P1 symbols, main L1 signaling cells and common PLPs are repeated over all RF channels forming the TFS structure. On the contrary, Type 1 PLPs are required to travel on the same $\mathrm{RF}$ channel during the $\mathrm{T} 2$ frame and the jumping can happen only between $\mathrm{T} 2$ frames. As a consequence, the addressing of PLPs data cells is not straightforward, if compared to the single RF channel DVB-T2 profile. The informative Annex $\mathrm{E}$ of the DVB-T2 specification [2] introduces the overall T2frame structure and distribution of PLP by type when using TFS.

With TFS many statistically multiplexed services can be therefore transmitted over more than one RF channel. Hence, a system performance improvement might be expected. Priori, two independent gains can be defined with TFS: Statistical multiplexing gain derived from the available larger "virtual channel" and a network planning coverage gain.

\section{B. Statistical Multiplexing Gain}

In [37] a model for a variable bit rate video traffic is proposed to study the performance of statistical multiplexing in a DVB-T2 system. The authors state that the TFS profile introduced in DVB-T2 in conjunction with StatMux can provide a valuable performance in terms of bandwidth efficiency. The potential benefits of TFS in terms of StatMux gain are also addressed in [38] where a rough approximation of the potential gain of TFS due to the increase of available services in the transmitted signal is also presented. For instance, provided estimations show a virtual bit rate increment that ranges the $21-25 \%$ when using 3 and 6 RF channels respectively with TFS.

\section{Network planning Gain}

The multiplexing of a PLP over multiple (not necessarily adjacent) channels has additional benefits. Without TFS the coverage area of a set of multiplexes at a given location is mainly limited by the multiplex with lowest signal strength. On the other hand, with TFS the overall reception of the TFS $\mathrm{RF}$ multiplex can be seen as the average signal strength of the RF channels involved in TFS. Under this assumption, the available frequency diversity in TFS provides a valuable gain margin compared to the traditional single profile. In [38] the main factors involved in the TFS network planing gain are outlined:

- TFS coverage gain: For given DVB-T2 network infrastructure, the ERP on multiple RF frequencies will have an impact on the received signal level due to the frequencydependency of several factors like the transmitter antenna diagram, reception environment and receiving antenna efficiency.

- TFS interference gain: TFS can provide robustness against interference from other transmitters.

- TFS can also provided robustness against hard channel reception conditions specially for portable and mobile reception.

Nevertheless, there are several factors that limit nowadays the adoption of TFS. The advantages of TFS are achieved at the expense of increasing receiver implementation complexity. The DVB-T2 TFS profile would required at least two tuners and a frequency hopping scheme. Restriction regarding the minimum hopping time between frequency channels is also a challenge to be addressed. Moreover, there are also regulatory aspects that may limit the current development of TFS. The lack of DVB-T2 receivers available in the market has so far limited the related research activities around the practical performance of TFS in DVB-T2. However, there have been some preliminary approaches that address the potential benefits of TFS.

The main research activities have been focused on two complementary approaches: field strength measurements over current available terrestrial broadcast infrastructures and system level simulations. In [38] [39] [40] empirically collected TFS network coverage gains are presented. The results imply network planning gain margins that go from $2.6 \mathrm{~dB}$ to $5.8 \mathrm{~dB}$ derived from the received signal strength in current DTT infrastructures at different RF channels in the UHF bands. Another set of results are provided by means of system levels simulations: Leaving aside the influence of synchronization and equalization stages in DVB-T2, in [41] authors presents the potential benefits of DVB-T2 TFS. Finally in [42] preliminary performance results for a complete DVB-T2 TFS software based receiver in a mobile scenario are also presented.

\section{CONClusions}

Since it was launched, DVB-T2 has shown that it is an extremely flexible and very functional system. The huge amount of configuration options allows the broadcasters to configure 
the system in the best way to maximize the robustness and capacity according to their intended reception scenarios.

One of the drawbacks of this flexibility is the difficulty to select the optimal configuration. In this paper the effects of some of the options have been presented according to simulations, laboratory test and field trials.

The configuration of the system for receiving fixed services with roof top antenna is the simplest one and the objective is to maximize the capacity at the lowest possible $\mathrm{C} / \mathrm{N}$ value. The results of field trials showed that the implementation loses are in the range of 1.4 to $2.5 \mathrm{~dB}$ for Gaussian and Rician channels.

In the case of mobile and portable reception many factors are present, like Doppler spread, delay spread or power variations. The results of the available tests using the configuration options appropriate to mobile performance have been presented. It has been shown that mobile reception can be achieved even with high FFT sizes. Also the possibility of using both DVB-T2 and T2-Lite profiles sharing the same RF channel has been tested and the efficiency gain has been quantified.

Regarding the performance of the most advanced options, MISO and TFS have shown that some additional gain might be achieved. Results provided so far show that the MISO gain margins available in DVB-T2 are mainly limited to the transmitter overlapping areas of an SFN. The MISO gain has proved to be significant when the power imbalance between MISO groups of the SFN network is small. However, results show as well that degradation might also happen using MISO in the non transmitter overlapping areas of an SFN where the power imbalance between MISO groups is large.

TFS is a different case, as it is not mandatory that a DVB-T2 receiver should work when TFS is used. It implies a more complex hardware and network design, but the preliminary studies currently available have shown that TFS can provide a valuable gain.

At the present time DVB-T2 has been commercially launched or officially adopted in more than 50 countries worldwide. Some of them, like India, Indonesia or Russia, are highly populated and the total population of those DVB-T2 countries is almost three billion people. This should lead to an important growth in the DVB-T2 equipment manufacturing industry and consequently to an increasing availability of low cost receivers. This is a key factor to ensure the success of any new broadcasting standard.

\section{REFERENCES}

[1] DVB, "Commercial requirement for DVB-T2," DVB, Blue Book A114, Apr. 2007.

[2] Digital Video Broadcasting (DVB); Frame structure channel coding and modulation for a second generation digital terrestrial television broadcasting system (DVB-T2), ETSI Std. EN 302755 V1.3.1, Apr. 2012.

[3] Digital Video Broadcasting (DVB); Modulator Interface (T2-MI) for a second generation digital terrestrial television broadcasting system (DVB-T2), ETSI Technical Specification TS 102773 V1.3.1, Jan. 2012.

[4] J. Cantillo, B. Collini-Nocker, U. De Bie, O. Del Rio, G. Fairhurst, A. Jahn, and R. Rinaldo, "GSE: A flexible, yet efficient, encapsulation for IP over DVB-S2 continuous generic streams," International Journal of Satellite Communications and Networking, vol. 26, no. 3, pp. 231-250, 2008. [Online]. Available: http://dx.doi.org/10.1002/sat.915
[5] T. Richardson, M. Shokrollahi, and R. Urbanke, "Design of capacityapproaching irregular low-density parity-check codes," Information Theory, IEEE Transactions on, vol. 47, no. 2, pp. 619-637, 2001.

[6] T. Richardson, "Error floors of LDPC codes," in Proceedings of the annual Allerton conference on communication control and computing, vol. 41, no. 3. The University; 1998, 2003, pp. 1426-1435.

[7] Digital Video Broadcasting (DVB); Implementation guidelines for a second generation digital terrestrial television broadcasting system (DVB-T2), ETSI Technical Specification TS 102831 V1.1.1, Oct. 2010.

[8] K. Boulle and J. Belfiore, "Modulation schemes designed for the Rayleigh channel," Proc. CISS92, pp. 288-293, 1992.

[9] C. Nour and C. Douillard, "Rotated QAM Constellations to Improve BICM Performance for DVB-T2," in Spread Spectrum Techniques and Applications, 2008. ISSSTA '08. IEEE 10th International Symposium on, Aug. 2008, pp. 354-359.

[10] D. Perez-Calderón, C. Oria, J. García, P. López, V. Baena, and I. Lacadena, "Rotated constellations for DVB-T2," in XXIV Conference on Design of Circuits and Integrated Systems, DCIS2009, vol. 1, Nov. 2009, pp. $128-133$.

[11] D. Perez-Calderon, V. Baena-Lecuyer, A. Oria, P. Lopez, and J. Doblado, "Simplified Rotated Constellation Demapper for Second Generation Terrestrial Digital Video Broadcasting," Broadcasting, IEEE Transactions on, vol. 59, no. 1, pp. 160-167, 2013.

[12] K. Kim, N. Basutkar, K. Bae, P. Xue, and H. Yang, "One-Dimensional Soft-Demapping Algorithms for Rotated QAM and Software Implementation on DSP," Signal Processing, IEEE Transactions on, vol. 61, no. 15 , pp. 3918-3930, 2013.

[13] S. Tomasin and M. Butussi, "Low Complexity Demapping of Rotated and Cyclic Q Delayed Constellations for DVB-T2," Wireless Communications Letters, IEEE, vol. 1, no. 2, pp. 81-84, 2012.

[14] V. Mignone and A. Morello, "CD3-OFDM: A novel demodulation scheme for fixed and mobile receivers," Communications, IEEE Transactions on, vol. 44, no. 9, pp. 1144-1151, 1996.

[15] A. Vigato, S. Tomasin, L. Vangelista, V. Mignone, N. Benvenuto, and A. Morello, "Coded Decision Directed Demodulation for Second Generation Digital Video Broadcasting Standard," Broadcasting, IEEE Transactions on, vol. 55, no. 3, pp. $607-615$, sept. 2009.

[16] B. Krongold and D. Jones, "PAR reduction in OFDM via active constellation extension," Broadcasting, IEEE Transactions on, vol. 49, no. 3, pp. 258-268, 2003.

[17] J. Tellado and J. Cioffi, "Efficient algorithms for reducing PAR in multicarrier systems," in Information Theory, 1998. Proceedings. 1998 IEEE International Symposium on, Aug. 1998, p. 191.

[18] J. G. Doblado, V. Baena, A. C. Oria, D. Perez-Calderon, and P. Lopez, "Coarse time synchronisation for DVB-T2," Electronics Letters, vol. 46, no. 11, pp. 797-799, 2010.

[19] M. Rotoloni, S. Tomasin, and L. Vangelista, "On correlation-based synchronization for DVB-T2," Communications Letters, IEEE, vol. 14 , no. 3, pp. 248-250, 2010.

[20] T. Jokela, M. Tupala, and J. Paavola, "Analysis of Physical Layer Signaling Transmission in DVB-T2 Systems," Broadcasting, IEEE Transactions on, vol. 56, no. 3, pp. 410-417, 2010.

[21] Frequency and Network Planning Aspects of DVB-T2, European Broadcasting Union Status Rep. EBU-TECH 3348, Version 2.0, May 2012.

[22] "NGH-PH.1 LAB TEST REPORT," Engines project deliverable D11.2, Dec. 2012.

[23] I. Eizmendi, G. Prieto, G. Berjon-Eriz, I. Landa, and M. Velez, "Empirical DVB-T2 Thresholds for Fixed Reception," Broadcasting, IEEE Transactions on, vol. 59, no. 2, pp. 306-316, 2013.

[24] N. Aurala, A. Vainisto, and R. Ekman, "Experiments with Rotated Constellation in DVB-T2," in International Broadcasting Convention (IBC 2012), Sep. 2012.

[25] I. Eizmendi, G. Prieto, G. Berjon-Eriz, M. Velez, A. Arrinda, and P. Angueira, "DVB-T2 performance in presence of multipath laboratory tests," in Broadband Multimedia Systems and Broadcasting (BMSB), 2011 IEEE International Symposium on. IEEE, Jun. 2011, pp. 1-6.

[26] D. Gozalvez, D. Gomez-Barquero, D. Vargas, and N. Cardona, "Time Diversity in Mobile DVB-T2 Systems," Broadcasting, IEEE Transactions on, vol. 57, no. 3, pp. 617-628, 2011.

[27] D. Gómez-Barquero, Next Generation Mobile Broadcast Systems. CRC Press, 2013.

[28] M. Slimani, J. Robert, and el al, "Results of the DVB-T2 Field Trial in Germany," Broadcasting, IEEE Transactions on, paper in process.

[29] T. Lang, "Terrestrik der Zukunft: Zukunft der Terrestrik Projektbericht DVB-T2 Norddeutschland," Tech. Rep. Shaker Verlag Aachen, 2012. 
[30] M. Slimani, J. Robert, and J. Zoellner, "A software-based mobile DVB-T2 measurement receiver," in Broadband Multimedia Systems and Broadcasting (BMSB), 2012 IEEE International Symposium on, Jun. 2012, pp. 1-6.

[31] C. Regueiro, G. Berjon-Eriz, I. Perez de Albeniz, I. Eizmendi, G. Prieto, and M. Velez, "DVB-T2 field trials results for portable indoor reception using T2-Lite and multiple PLP," in Broadband Multimedia Systems and Broadcasting (BMSB), 2013 IEEE International Symposium on. IEEE, 2013, pp. 1-5

[32] S. Alamouti, "A simple transmit diversity technique for wireless communications," Selected Areas in Communications, IEEE Journal on, vol. 16 , no. 8, pp. 1451-1458, 1998.

[33] M. Tormos, C. Tanougast, P. Kasser, P. Bretillon, and A. Dandache, "Performance evaluation for different MISO - SFN network combinations for DVB-T2 standard," in Broadband Multimedia Systems and Broadcasting (BMSB), 2011 IEEE International Symposium on, 2011, pp. 1-4.

[34] M. Tormos, C. Tanougast, A. Dandache, P. Bretillon, and P. Kasser, "Experimental performance of mobile DVB-T2 in SFN and distributed MISO network," in Telecommunications (ICT), 2012 19th International Conference on, 2012, pp. 1-5.

[35] J. Qi, J. Robert, K. Chee, M. Slimani, and J. Zoellner, "DVB-T2 MISO field measurements and a calibrated coverage gain predictor," in Broadband Multimedia Systems and Broadcasting (BMSB), 2012 IEEE International Symposium on, 2012, pp. 1-6.

[36] J. Morgade, P. Angueira, A. Arrinda, R. Pfeffer, V. Steinmann, J. Frank, and R. Brugger, "SFN-SISO and SFN-MISO Gain Performance Analysis for DVB-T2 Network Planning," Broadcasting, IEEE Transactions on, vol. PP, no. 99, pp. 1-1, 2014.

[37] R. Mehdi, B. Imed, , and G. Moncef, "Statistical Time-Frequency Multiplexing of HD Video Traffic in DVB-T2," International Journal of Digital Multimedia Broadcasting, vol. 2009, p. 12, 2009.

[38] Frequency and Network Planning Aspects of DVB-T2, European Broadcasting Union Status Rep. EBU-TECH 3348, May 2011.

[39] D. Gozalvez, J. Lopez-Sanchez, D. Gomez-Barquero, J. Gimenez, and N. Cardona, "Combined Time and Space Diversity: Mobile Reception in DVB-T and DVB-T2 Systems," Vehicular Technology Magazine, IEEE, vol. 7, no. 4, pp. 114-121, 2012.

[40] J. Gimenez, D. Gozalvez, D. Gomez-Barquero, and N. Cardona, "Statistical model of signal strength imbalance between RF channels in DTT network," Electronics Letters, vol. 48, no. 12, pp. 731-732, 2012.

[41] M. Makni, J. Robert, and E. Stare, "Performance analysis of time frequency slicing," in Electronic Media Technology, CEMT, 2011 14th ITG Conference on, 2011, pp. 1-6.

[42] J. Morgade, P. Angueira, J. A. Arenas, A. Basterra, D. Plets, and W. Joseph, "A Software Based DVB-T2 Time Frequency Slicing Evaluation Framework," in Broadband Multimedia Systems and Broadcasting, BMSB. 2013 IEEE International Symposium on, 2013.

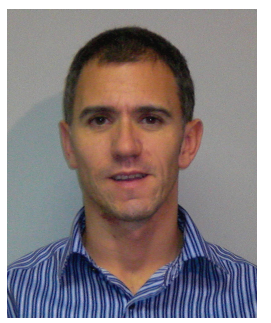

Iñaki Eizmendi received the M.S. and $\mathrm{PhD}$ in Telecommunications Engineering at the University of the Basque Country, Spain, in 1994 and 2012 respectively. He has worked as R\&D engineer in several companies. From 2003 he is with the TSR (Radiocommunications and Signal Processing) research group at the Communications Engineering Dpt. of the University of the Basque Country (UPV/EHU). $\mathrm{He}$ is an assistant professor in the same department.

His current research interests focus on new digital standards (DVB-T, DVB-T2, DVB-NGH).

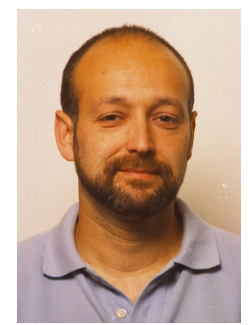

Manuel Vélez received the M.S. and $\mathrm{PhD}$ in Telecommunication Engineering at the University of the Basque Country (Spain) in 1993 and 2008 respectively. In 1995 he joined the Communications Engineering Dpt. of the UPV/EHU, where he is currently an assistant professor. He has been involved for 15 years in research projects evaluating radio and television broadcasting systems. His research interests are related to DVB-T2 and DVB-NGH, in the areas of software defined radio developments, performance evaluation.

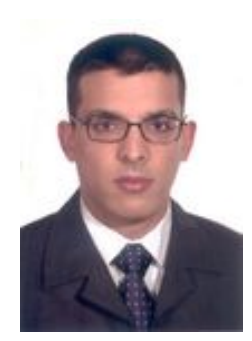

David Goḿez-Barquero received the double M.Sc. degree in telecommunications engineering from the Universitat Politècnica de València (UPV), Spain, and the University of Gävle, Sweden, in 2004, and the Ph.D. degree in telecommunications from UPV in 2009. During his doctoral studies, he was a Guest Researcher at the Royal Institute of Technology, Sweden, the University of Turku, Finland, and the Technical University of Braunschweig, Germany. He also did an internship at Ericsson Eurolab, Germany. From 2010 to 2011, he was a Post-Doctoral Guest Researcher at the Fraunhofer Heinrich Hertz Institute, Germany.

He is currently a Senior Researcher (Ramon y Cajal Fellow) at the Institute of Telecommunications and Multimedia Applications (iTEAM), UPV, where he leads a research group working on multimedia broadcasting, in particular on the optimization of 3GPP multimedia broadcast multicast services (MBMS) and digital video broadcasting (DVB) systems.

Since 2008, he has been actively participating in the European digital television standardization forum DVB. He participated in the validation of DVB-T2, and in the standardization processes of its mobile profile T2-Lite and its handheld evolution DVB-NGH. He also contributed to the DVB-T2 implementation guidelines, and co-edited the implementation guidelines for upper layer forward error correction. He was very involved in the promotion and adoption of DVB-T2 in Colombia, and since the second half of 2012 he has been working with the spectrum regulator on the spectrum and network planning and optimization of DVB-T2. Since then, he is also a Visiting Professor at the Sergio Arboleda University of Bogota, Colombia.

Dr. Gómez-Barquero is the Editor of the book "Next Generation Mobile Broadcasting" (CRC Press), and the Vice-Chairman of the Modulation and Coding Ad-Hoc Group of the ATSC 3.0 standardization process.

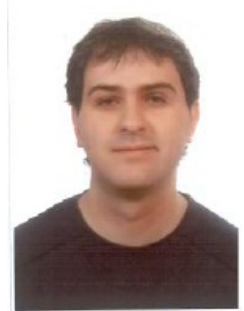

Javier Morgade received the B.Sc. and M.Sc. degrees in telecommunications engineering from the University of Basque Country, Spain, in 2006 and 2009. He is nowadays finalizing his PhD. In 2006 he joined the Radio Communications and Signal Processing research group (TSR) at the Department of Communications Engineering of the University of the Basque Country (UPV/EHU). Within the TSR group, he was involved in several research projects to evaluate digital terrestrial broadcast standards such as DVB-T/H (Digital Video Broadcasting) and DRM (Digital Radio Mondiale). In 2010, he was granted by the R\&D Council of the Basque Government (GV/EJ-BI09.65) to carry out his PhD in the field of Next Generation Broadcast Systems under the Department of Communications Engineering of the UPV/EHU. His current research interests include several aspects of service planning and signal processing for new broadcast technologies like DVB-T2 and DVB-NGH. Since Jan'14, he has joined Standards and Industry Affairs group at Samsung Electronics R\&D UK. 


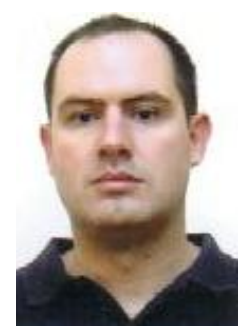

Vicente Baena-Lecuyer was born in Athis-Mons, France. He received the Telecommunication Engineering and $\mathrm{Ph} . \mathrm{D}$. degrees from the University of Seville, Seville, Spain, in 1997 and 2001, respectively. Since 1997, he has been with the Department of Electronic Engineering, Escuela Superior de Ingenieros, University of Seville. His current research interests include orthogonal frequency division multiplexing systems, especially digital video broadcasting systems.

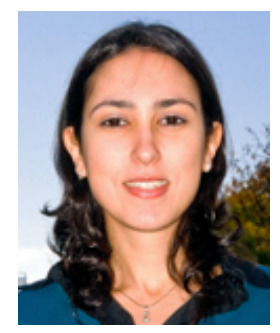

Mariem Slimani studied Electrical Engineering at Technische Universitaet Braunschweig, Germany. In her diploma thesis she evaluated the performance of Time Frequency Slicing for DVBs new system DVB-NGH. In October 2010 she became a researcher at the Institut fuer Nachrichtentechnik of Technische Universitaet Braunschweig working on the performance evaluation of the mobile reception of DVB-T2.

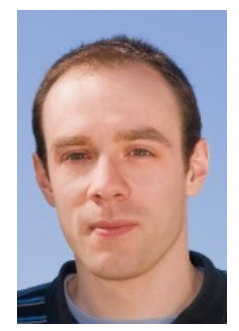

Jan Zoellner received his diploma degree in Computer Science and Communications Technology Engineering from Technische Universitaet Braunschweig in 2010. His diploma thesis resulted in the implementation of a DVB-C measurement receiver in MATLAB. Upon graduation he joined the Institut fuer Nachrichtentechnik at Technische Universitaet Braunschweig where he was involved in the development of DVB-NGH. He currently chairs DVB's Study Mission on co-operative spectrum use. 